JOURNAL OF THE

AMERICAN MATHEMATICAL SOCIETY

Volume 13, Number 1, Pages 83-99

S 0894-0347(99)00315-X

Article electronically published on August 20, 1999

\title{
ALGEBRAIC FAMILIES OF NONZERO ELEMENTS OF SHAFAREVICH-TATE GROUPS
}

\author{
JEAN-LOUIS COLLIOT-THÉLÈNE AND BJORN POONEN
}

\section{INTRODUCTION}

Around 1940, Lind [Lin] and (independently, but shortly later) Reichardt [Re] discovered that some genus 1 curves over $\mathbf{Q}$, such as

$$
2 y^{2}=1-17 x^{4}
$$

violate the Hasse principle; i.e., there exist $x, y \in \mathbf{R}$ satisfying the equation, and for each prime $p$ there exist $x, y \in \mathbf{Q}_{p}$ satisfying the equation, but there do not exist $x, y \in \mathbf{Q}$ satisfying the equation. In fact, even the projective nonsingular model has no rational points.

We address the question of constructing algebraic families of such examples. Can one find an equation in $x$ and $y$ whose coefficients are rational functions of a parameter $t$, such that specializing $t$ to any rational number results in a genus 1 curve violating the Hasse principle? The answer is yes, even if we impose a nontriviality condition; this is the content of our Theorem 1.2.

Any genus 1 curve $X$ is a torsor for its Jacobian $E$. Here $E$ is an elliptic curve: a genus 1 curve with a rational point. If $X$ violates the Hasse principle, then $X$ moreover represents a nonzero element of the Shafarevich-Tate group $\amalg(E)$. We may relax our conditions by asking for families of torsors of abelian varieties. In this case we can find a family with stronger properties: one in which specializing the parameter to any number $\alpha \in \overline{\mathbf{Q}}$ of odd degree over $\mathbf{Q}$ results in a torsor of an abelian variety over $\mathbf{Q}(\alpha)$ violating the Hasse principle. A precise version of this result follows. (Throughout this paper, $\mathbf{P}^{1}$ unadorned denotes the projective line over Q.)

Theorem 1.1. There exists an open subscheme $U$ of $\mathbf{P}^{1}$ containing all closed points of odd degree, and there exist smooth projective geometrically integral varieties $\mathcal{A}$ and $\mathcal{X}$ over $\mathbf{Q}$ equipped with dominant morphisms $\pi_{\mathcal{A}}$ and $\pi_{\mathcal{X}}$ to $\mathbf{P}^{1}$ such that

(1) $\mathcal{A}_{U}:=\pi_{\mathcal{A}}^{-1}(U)$ is an abelian scheme of relative dimension 2 over $U$.

Received by the editors January 8, 1999 and, in revised form, June 9, 1999.

1991 Mathematics Subject Classification. Primary 11G10; Secondary 11G30, 11G35, 14H40, $14 \mathrm{~J} 27$.

Key words and phrases. Shafarevich-Tate group, Brauer-Manin obstruction, Hasse principle, cubic surface, Cassels-Tate pairing, Lefschetz pencil.

Most of the research for this paper was done while the authors were both enjoying the hospitality of the Isaac Newton Institute, Cambridge, England. The first author is a researcher at C.N.R.S. The second author is partially supported by NSF grant DMS-9801104, a Sloan Fellowship, and a Packard Fellowship. 
(2) $\mathcal{X}_{U}:=\pi_{\mathcal{X}}^{-1}(U)$ is an $\mathcal{A}_{U}$-torsor over $U$.

(3) $\pi_{\mathcal{X}}\left(\mathcal{X}\left(k_{v}\right)\right)=\mathbf{P}^{1}\left(k_{v}\right)$ for every local field $k_{v} \supset \mathbf{Q}$ (archimedean or not).

(4) $\mathcal{X}$ has no zero-cycle of odd degree over $\mathbf{Q}$.

(5) $\mathcal{A}$ is a nonconstant family (i.e., there exist $u, v \in U(\overline{\mathbf{Q}})$ such that the fibers $\mathcal{A}_{u}$ and $\mathcal{A}_{v}$ are not isomorphic over $\left.\overline{\mathbf{Q}}\right)$.

(6) The generic fiber of $\mathcal{A} \rightarrow \mathbf{P}^{1}$ is absolutely simple (i.e., simple over $\overline{\mathbf{Q}(t)}$ ).

Remarks. 1. Conditions (1) through (4) imply that for each closed point $s \in U$ of odd degree (in particular, for all $s \in \mathbf{P}^{1}(\mathbf{Q})$ ), the fiber $\mathcal{X}_{s}$ represents a nonzero element of the Shafarevich-Tate group $\amalg\left(\mathcal{A}_{s}\right)$.

2. Let $E$ be an elliptic curve over $\mathbf{Q}$, and suppose that $C$ is a genus 1 curve over $\mathbf{Q}$ representing an element of $\amalg(E)$ whose order is even. Let $\mathcal{B}$ be a smooth projective geometrically integral variety over $\mathbf{Q}$ equipped with a morphism $\pi_{\mathcal{B}}: \mathcal{B} \rightarrow \mathbf{P}^{1}$ making $\pi_{\mathcal{B}}^{-1}(U)$ a nonconstant abelian scheme over $U, U$ being as above. Then $\mathcal{A}:=E \times_{\mathbf{Q}} \mathcal{B}$ and $\mathcal{X}:=C \times_{\mathbf{Q}} \mathcal{B}$, equipped with the morphisms to $\mathbf{P}^{1}$ obtained by composing the second projection with $\mathcal{B} \rightarrow \mathbf{P}^{1}$, satisfy (1) through (5). Condition (6) is designed to rule out such "trivial" examples.

The varieties $\mathcal{A}$ and $\mathcal{X}$ promised by the theorem will be smooth compactifications (over $\mathbf{Q}$ ) of $\mathbf{P} \mathbf{c}_{\mathcal{C} / \mathbf{P}^{1}}^{0}$ and $\mathbf{P i c} \mathbf{C}_{\mathcal{C} / \mathbf{P}^{1}}^{1}$ for a relative curve $\mathcal{C} \rightarrow \mathbf{P}^{1}$ of genus 2. (For the theory of the relative Picard functor, we refer the reader to [BLR].) The key ingredient which will let us prove the nonexistence of rational points of $\mathcal{X}$ over fields of odd degree (condition (4)) is the formula from [PS] which gives the value of the Cassels-Tate pairing when the torsor $\mathbf{P i c}{ }^{g-1}$ of $\mathbf{P i c}{ }^{0}$ for a genus $g$ curve over a global field is paired with itself. Using that same formula, we will show that the nonexistence of zero-cycles of degree one on $\mathcal{X}$ can be explained by a Brauer-Manin obstruction.

As mentioned at the outset, we also have an example of relative dimension 1. Here we control fibers only above rational points, not above all points of odd degree.

Theorem 1.2. There exist an open subscheme $U$ of $\mathbf{P}^{1}$ containing $\mathbf{P}^{1}(\mathbf{Q})$ and smooth projective geometrically integral varieties $\mathcal{E}$ and $\mathcal{X}$ over $\mathbf{Q}$ equipped with dominant morphisms $\pi_{\mathcal{E}}$ and $\pi_{\mathcal{X}}$ to $\mathbf{P}^{1}$ such that

(1) $\mathcal{X}_{U} \rightarrow U$ is a proper smooth family whose fibers are geometrically integral curves of genus 1 .

(2) $\mathcal{E}_{U}=\mathbf{P i c}_{\mathcal{X}_{U} / U}^{0}$, so $\mathcal{E}$ is an elliptic surface over $\mathbf{P}^{1}$, smooth above $U$.

(3) $\pi_{\mathcal{X}}\left(\mathcal{X}\left(\mathbf{Q}_{p}\right)\right)=\mathbf{P}^{1}\left(\mathbf{Q}_{p}\right)$ for all $p \leq \infty$.

(4) $\mathcal{X}(\mathbf{Q})$ is empty.

(5) The $j$-invariant of $\mathcal{E}_{U} \rightarrow U$ is a nonconstant function on $U$.

The proof of this begins by taking a Lefschetz pencil in a cubic surface violating the Hasse principle. A suitable base change of this will give us $\mathcal{X} \rightarrow \mathbf{P}^{1}$. When the failure of the Hasse principle for the cubic surface is due to a Brauer-Manin obstruction, ${ }^{1}$ we can construct a second family $\mathcal{Y}_{U} \rightarrow U$ of genus 1 curves such that $\mathbf{P i c}_{\mathcal{Y}_{U} / U}^{0}=\mathcal{E}_{U}$, the analogue of (3) holds, and the Cassels-Tate pairing satisfies $\left\langle\mathcal{X}_{t}, \mathcal{Y}_{t}\right\rangle=1 / 3$ for all $t \in U(\mathbf{Q})$. While proving the last statement, we are led to prove an auxiliary result (Lemma 3.4 ) which may be of independent interest:

\footnotetext{
${ }^{1}$ It has been conjectured that the Brauer-Manin obstruction is the only obstruction to the Hasse principle for cubic surfaces. In any case, there are examples of cubic surfaces violating the Hasse principle because of such an obstruction. See 2.5 and 3.1 below.
} 
if there is a Brauer-Manin obstruction to the Hasse principle for a smooth cubic surface $V$, then only one element $\alpha \in \operatorname{Br}(V)$ is needed to create the obstruction.

Throughout this paper, étale cohomology of a scheme $X$ with values in a sheaf $\mathcal{F}$ is denoted $\mathrm{H}_{\text {ét }}^{*}(X, \mathcal{F})$. The notation for the Galois cohomology of (the spectrum of) a field $k$ will simply be $\mathrm{H}^{*}(k, \mathcal{F})$. The (cohomological) Brauer group $\operatorname{Br}(X)$ of a scheme $X$ is the group $\mathrm{H}_{\text {ét }}^{2}\left(X, \mathbf{G}_{m}\right)$. The Shafarevich-Tate group $\amalg(A)$ of an abelian variety $A$ over a number field $k$ is defined as the kernel of the natural map $\mathrm{H}^{1}(k, A) \rightarrow \prod_{v} \mathrm{H}^{1}\left(k_{v}, A\right)$, where the product is over all places $v$ of $k$, and $k_{v}$ denotes the completion. If $k$ is a field, then $\bar{k}$ denotes an algebraic closure of $k$, and if $X$ is a $k$-scheme, then $\bar{X}:=X \times_{k} \bar{k}$. Given an integral (i.e., reduced and irreducible) $k$-variety, we write $k(X)$ for the function field of $X$. If $X / k$ is geometrically integral, we denote by $\bar{k}(X)$ the function field of $\bar{X}$.

\section{A FAMILY OF ABELIAN SURFACES}

In this section we prove Theorem 1.1.

2.1. Minimal models of hyperelliptic curves. We need a simple lemma describing the minimal model of certain hyperelliptic curves. Recall $[\mathrm{DM}]$ that a stable curve over a base $S$ is a proper flat morphism $\mathcal{C} \rightarrow S$ whose geometric fibers are reduced, connected, 1 -dimensional schemes $\mathcal{C}_{s}$ such that $\mathcal{C}_{s}$ has only ordinary double points, and any nonsingular rational component of $\mathcal{C}_{s}$ meets the other components in more than 2 points.

Lemma 2.1. Let $R$ be a Dedekind domain with $2 \in R^{*}$, and let $K$ be its fraction field. Let $f(x, z) \in R[x, z]$ be homogeneous of even degree $2 n \geq 4$, and assume that its discriminant generates a squarefree ideal of $R$. Let $C / K$ be the smooth projective model of the affine curve defined by $y^{2}=f(x, 1)$ over $K$. Then the minimal proper regular model $\mathcal{C} / R$ of $C$ is stable and can be obtained by glueing the affine curves $y^{2}=f(x, 1)$ and $Y^{2}=f(1, z)$ over $R$ along the open subsets where $x \neq 0$ and $z \neq 0$ respectively, using the identifications $z=x^{-1}$ and $Y=x^{-n} y$. In particular, its geometric fibers are integral.

Proof. The model $\mathcal{C}$ described is finite over $\mathbf{P}_{R}^{1}$ (which manifests itself here as two copies of $\mathbf{A}_{R}^{1}$ with variables $x, z$, glued along $x \neq 0$ and $z \neq 0$ using the identification $z=x^{-1}$ ), so $\mathcal{C}$ is proper over $R$. The regularity and stability follow from Corollaire 6 and Remarque 18 in [Liu]. The integrality of the fibers follows from the explicit construction, and implies that $\mathcal{C}$ is the minimal model.

2.2. The relative curve. Let $C$ be the smooth projective model of the curve

$$
y^{2}=-\left[x^{6}+x^{5}+t^{5} x+\left(8 t^{6}+7\right)\right]
$$

over $\mathbf{Q}(t)$. Let $f_{t}(x) \in \mathbf{Q}[t][x]$ denote the right hand side of (2.1). PARI shows that the discriminant $\Delta(t)$ of $f_{t}(x)$ with respect to $x$ is an irreducible polynomial of degree 30 in $\mathbf{Q}[t]$. In particular $\Delta(t) \not \equiv 0$, so $C$ is of genus 2. Let $s_{0} \in \mathbf{A}_{\mathbf{Q}}^{1} \subset \mathbf{P}^{1}$ be the closed point corresponding to $\Delta(t)$, and let $U=\mathbf{P}^{1} \backslash\left\{s_{0}\right\}$.

Since $\Delta(t)$ is squarefree, the minimal model $\mathcal{C}^{0}$ over $\operatorname{Spec} \mathbf{Q}[t]$ is described by Lemma 2.1. Let $T=t^{-1}$. Then $C$ is birational over $\mathbf{Q}(t)$ to the affine curve

$$
Y^{2}=-\left[X^{6}+T X^{5}+X+\left(8+7 T^{6}\right)\right]
$$

via the change of variables $X=x / t$ and $Y=y / t^{3}$. The minimal model $\mathcal{C}^{1}$ over $\operatorname{Spec} \mathbf{Q}[T]$ is given by Lemma 2.1 again. Glueing $\mathcal{C}^{0}$ and $\mathcal{C}^{1}$ gives the minimal model 
$\mathcal{C} \rightarrow \mathbf{P}^{1}$. Then $\mathcal{C} \rightarrow \mathbf{P}^{1}$ is stable, and has good reduction over $U$, since this can be checked locally on the base. Moreover $\mathcal{C}$ is smooth and proper over $\mathbf{Q}$.

2.3. Local points on the fibers. In what follows, we will specialize $\mathcal{C}$ at points in $\mathbf{P}^{1}(k)$ where $k$ is a field containing $\mathbf{Q}$. Although $t$ above denoted an indeterminate, we will abuse notation below by letting $t$ denote also the specialized value in $k \cup\{\infty\}$, and the corresponding $k$-valued point Spec $k \rightarrow \mathbf{P}^{1}$. If $t \neq \infty$ (resp. $\left.t \neq 0\right)$, then the fiber $\mathcal{C}_{t}$ is birational to the curve over $k$ given by the equation (2.1) (resp. (2.2)).

Lemma 2.2. (1) If $t \in U(\mathbf{R})$, then $\mathcal{C}_{t}$ has no (real) divisor of degree 1.

(2) If $k$ is a finite extension of $\mathbf{Q}_{p}$ for some finite prime $p$, and if $t \in U(k)$, then $\mathcal{C}_{t}(k) \neq \emptyset$.

Proof. For $t, x \in \mathbf{R}$, the weighted AM-GM inequality gives

$$
\frac{1}{6} x^{6}+\frac{5}{6} t^{6} \geq\left|t^{5} x\right| \geq-t^{5} x \quad \text { and } \quad \frac{5}{6} x^{6}+\frac{1}{6} \geq\left|x^{5}\right| \geq-x^{5} .
$$

Combining these shows that

$$
x^{6}+x^{5}+t^{5} x+\left(8 t^{6}+7\right)>0 .
$$

Thus $\mathcal{C}$ has a dense open subscheme with no real points. But $\mathcal{C} \times{ }_{\mathbf{Q}} \mathbf{R}$ is smooth over $\mathbf{R}$, so $\mathcal{C}(\mathbf{R})=\emptyset$. Hence $\mathcal{C}$ and $\mathcal{C}_{t}$ for $t \in U(\mathbf{R})$ have no real zero-cycles of odd degree.

From now on, we assume $t \in U(k)$ where $\left[k: \mathbf{Q}_{p}\right]<\infty$, and we let $v_{p}$ be the $p$-adic valuation on $k$, normalized so that $v_{p}(p)=1$. By convention, if $t=\infty$, then $v_{p}(t)=-\infty<0$. First suppose $p=2$. If $v_{2}(t)<0$, then Hensel's Lemma shows that (2.2) has a $k$-point with $Y=0$, and with $X$ near 0 . If $v_{2}(t) \geq 0$, then (2.1) has a $k$-point with $x=0$.

From now on, we assume $p$ is odd. Let $\mathbf{F}_{q}$ be the residue field of $k$. Suppose $v_{p}(t) \geq 0$. Then let $f(x) \in \mathbf{F}_{q}[x]$ denote the reduction of the right hand side of (2.1). If $f(x)$ is a square in $\overline{\mathbf{F}}_{p}[x]$, then equating coefficients of $x^{6}, x^{5}, x^{4}, x^{3}$ shows that the reduction would have to be $-\left(x^{3}+x^{2} / 2-x / 8+1 / 16\right)^{2}$, but equating the coefficients of $x^{2}, x^{1}, x^{0}$ gives the inconsistent system

$$
0=-5 / 64, \quad \bar{t}^{5}=-1 / 64, \quad 7+8 \bar{t}^{6}=1 / 256,
$$

of equations in $\overline{\mathbf{F}}_{p}$, where $\bar{t}$ denotes the reduction of $t$. Hence $f(x)$ is not the square of any polynomial in $\overline{\mathbf{F}}_{p}[x]$. If instead $v_{p}(t)<0$, then let $f(X)=-\left(X^{6}+X+8\right) \in$ $\mathbf{F}_{q}[X]$ be the reduction of the right hand side of (2.2), which again is not a square in $\overline{\mathbf{F}}_{p}[X]$.

In either case (no matter what the value of $v_{p}(t)$ ), write $f(x)=j(x)^{2} h(x)$ in $\mathbf{F}_{q}[x]$ with $h(x)$ squarefree, so by the previous paragraph $\operatorname{deg} h>0 .^{2}$ Any $\mathbf{F}_{q}$-point on $z^{2}=h(x)$ with $x$ finite and $j(x) \neq 0$ gives rise to a nonsingular $\mathbf{F}_{q}$-point on the affine curve $y^{2}=f(x)$, which can be lifted by Hensel's Lemma to prove $\mathcal{C}_{t}(k) \neq \emptyset$. Also, if there is a point at $\infty$ on (the projective nonsingular model of) $z^{2}=h(x)$ over $\mathbf{F}_{q}$, then there will be points on $\mathcal{C}_{t}$ at $\infty$. The number of points on $z^{2}=h(x)$ with $j(x)=0$ is at most $2 \operatorname{deg} j=6-\operatorname{deg} h=6-(2 g+2)=4-2 g$, where $g$ is the

\footnotetext{
${ }^{2}$ At this point we know that $y^{2}=f(x)$ has a multiplicity one component which is geometrically integral, so it follows automatically as in Proposition 3.1 in [vGY] or Lemma 15 in [PS] that $\mathcal{C}_{t}$ has a $k$-rational divisor of degree 1. In fact this would suffice for our application, except in Section 2.7, where it will be convenient to have the stronger result $\mathcal{C}_{t}(k) \neq \emptyset$.
} 
geometric genus of $z^{2}=h(x)$. Therefore by the Weil bound, we automatically find the desired point if

$$
(q+1-2 g \sqrt{q})-(4-2 g)>0
$$

for $g=0,1,2$. These hold for $q \geq 17$, so it remains to consider the cases $q=$ $3,5,7,9,11,13$. If $q \equiv 1(\bmod 4)$, then $\mathcal{C}_{t}$ has $k$-points at $\infty$. For each remaining $q$, we check by hand that for each possible residue class of $t$ (and for the case $T \equiv 0$ in (2.2)), there is still a nonsingular affine point on $y^{2}=f(x)$.

2.4. Components of the Picard scheme. Since $\mathcal{C}$ is a proper smooth surface over $\mathbf{Q}$, it is projective over $\mathbf{Q}$, and hence also projective over $\mathbf{P}^{1}$. Moreover the geometric fibers of $\mathcal{C} \rightarrow \mathbf{P}^{1}$ are integral. Hence we may apply $9.3 / 1$ from [BLR] to deduce that

1. The relative Picard functor $\mathbf{P i c _ { C } / \mathbf { P } ^ { 1 }}$ is represented by a separated and locally smooth $\mathbf{P}^{1}$-scheme.

2. $\mathbf{P i c}_{\mathcal{C} / \mathbf{P}^{1}}=\bigsqcup_{n \in \mathbf{Z}} \mathbf{P} \mathbf{c}_{\mathcal{C} / \mathbf{P}^{1}}^{n}$, where $\mathbf{P i c} \mathbf{C}_{\mathcal{C} / \mathbf{P}^{1}}^{n}$ denotes the open and closed subscheme of $\mathbf{P i c} \mathbf{C}_{\mathcal{C} / \mathbf{P}^{1}}$ consisting of all line bundles of degree $n$.

3. For each $n \in \mathbf{Z}, \mathbf{P i c}_{\mathcal{C} / \mathbf{P}^{1}}^{n}$ is a torsor for $\mathbf{P} \mathbf{c}_{\mathcal{C} / \mathbf{P}^{1}}^{0}$ and is quasi-projective.

Let $\tilde{\mathcal{A}}:=\mathbf{P} \mathbf{i c}_{\mathcal{C} / \mathbf{P}^{1}}^{0}$ and let $\tilde{\mathcal{X}}:=\mathbf{P i c}_{\mathcal{C} / \mathbf{P}^{1}}^{1}$. For any $\mathbf{P}^{1}$-scheme $\pi: Z \rightarrow \mathbf{P}^{1}$, let $Z_{U}$ denote $\pi^{-1}(U)$. Then $\tilde{\mathcal{A}}_{U}$ is an abelian $U$-scheme, by $9.4 / 4$ from [BLR]. Resolution of singularities gives us $\mathbf{P}^{1}$-schemes $\pi_{\mathcal{A}}: \mathcal{A} \rightarrow \mathbf{P}^{1}$ and $\pi_{\mathcal{X}}: \mathcal{X} \rightarrow \mathbf{P}^{1}$ such that

1. $\mathcal{A}$ and $\mathcal{X}$ are smooth and projective over $\mathbf{Q}$.

2. $\mathcal{A}_{U} \cong \tilde{\mathcal{A}}_{U}$ and $\mathcal{X}_{U} \cong \tilde{\mathcal{X}}_{U}$ as $U$-schemes.

We now verify that $\mathcal{A}$ and $\mathcal{X}$ satisfy the conditions of Theorem 1.1. Conditions (1) and (2) are clear from the construction. The following lemma applied to the smooth fibers $\mathcal{C}_{t}$ shows that $\pi_{\mathcal{X}}\left(\mathcal{X}\left(k_{v}\right)\right) \supset U\left(k_{v}\right)$, and condition $(3)$ then follows from compactness of $\pi_{\mathcal{X}}\left(\mathcal{X}\left(k_{v}\right)\right)$.

Lemma 2.3. Let $k$ be a local field (i.e., a finite extension of $\mathbf{R}, \mathbf{Q}_{p}$, or $\mathbf{F}_{p}((t))$ ), and let $\bar{k}$ denote a separable closure of $k$. Let $X$ be a smooth projective geometrically integral curve over $k$ of genus $g$, and let $\bar{X}=X \times_{k} \bar{k}$. Then there exists an element of degree $g-1$ in $\operatorname{Pic}(\bar{X})$ which is stable under $\operatorname{Gal}(\bar{k} / k)$.

Proof. This is a consequence of Tate local duality. See Theorem 7 on p. 133 of [Lic] for the case where $k$ is a finite extension of $\mathbf{Q}_{p}$, or Section 4 of [PS] for the general case. The real case is related to W.-D. Geyer's modern version of work of Weichold and Witt; see [Sc], Thm. (20.1.5.1), p. 221.

We now prove condition (4). Suppose condition (4) fails, so that $\mathcal{X}$ has a closed point of odd degree. Its image under $\pi$ is a closed point of $\mathbf{P}^{1}$ of odd degree. Since $U$ contains all points of $\mathbf{P}^{1}$ of odd degree, this closed point corresponds to some $t \in U(k)$ with $[k: \mathbf{Q}]$ odd, such that the fiber $\mathcal{X}_{t}$ has a closed point of odd degree over $k$. Since $\mathcal{C}_{t}$ admits a 2-to-1 map to $\mathbf{P}_{k}^{1}$, it possesses a point in a quadratic extension of $k$, hence $\mathcal{X}_{t}=\mathbf{P i c}_{\mathcal{C}_{t} / k}^{1}$ also possesses such a point. Thus the principal homogeneous space $\mathcal{X}_{t}=\mathbf{P i c}_{\mathcal{C}_{t} / k}^{1}$ of $\mathcal{A}_{t}=\mathbf{P i c}_{\mathcal{C}_{t} / k}^{0}$ is annihilated by coprime integers, hence $\mathcal{X}_{t}$ is trivial.

On the other hand, we will show that $\mathcal{X}_{t}$ cannot be trivial because of the following, which is Corollary 12 in [PS] (the assertion $c \in \amalg(A)$ is Lemma 2.3 above). 
Lemma 2.4. Let $k$ be a global field (i.e., a finite extension of $\mathbf{Q}$ or $\mathbf{F}_{p}(t)$ ). Let $C$ be a smooth projective geometrically integral curve over $k$. Let $A$ be the canonically polarized jacobian of $C$, and let $c$ be the class of $\mathbf{P i c}_{C / k}^{g-1}$ in $\mathrm{H}^{1}(k, A)$. Then $c \in \amalg(A)$, and the Cassels-Tate pairing satisfies $\langle c, c\rangle=N / 2 \in \mathbf{Q} / \mathbf{Z}$, where $N$ is the number of places $v$ of $k$ for which $C$ has no $k_{v}$-rational divisor of degree $g-1$.

Let us apply this to the fiber $C=\mathcal{C}_{t}$. Then $g=2, c$ is the class of $\mathcal{X}_{t}$, and $N$ equals the number of real places of $k$, by Lemma 2.2. Since $[k: \mathbf{Q}]$ is odd, $N$ is odd. Thus $\langle c, c\rangle \neq 0$, so $c \neq 0$; i.e., $\mathcal{X}_{t}$ is nontrivial in $\amalg\left(\mathcal{A}_{t}\right)$. This contradiction proves condition (4).

We next prove condition (5). The curve $\mathcal{C}_{U}$ is projective and smooth over $U$. There is an associated $\mathbf{Q}$-morphism $f$ from $U$ to the coarse moduli space $M_{2}$ of smooth curves of genus 2, and the jacobian functor yields a $\mathbf{Q}$-morphism $j: M_{2} \rightarrow \mathcal{A}_{2}$ to the coarse moduli space of principally polarized abelian surfaces over $\mathbf{Q}$. By Torelli's theorem, the map $j$ is injective over algebraically closed fields [MFK, p. 143]. Thus to prove (5), it is enough to show that $f$ is not constant. Suppose $f$ is constant. Let $R$ be the completion of the local $\operatorname{ring}$ of $\mathbf{P}^{1}$ at the closed point where $\mathcal{C} / \mathbf{P}^{1}$ has bad reduction, and let $K$ be the fraction field of $R$. Since $f$ is constant, there exists a finite field extension $L / K$ such that $\mathcal{C} \times \mathbf{P}^{1} L$ is $L$-isomorphic to a constant curve. The latter has good reduction over the ring of integers of $L$, but for a stable curve the property of having good reduction does not depend on the field extension, so the stable curve $\mathcal{C} \times{ }_{\mathbf{P}^{1}} R$ must be smooth. This is a contradiction.

Finally we must show that the generic fiber of $\mathcal{A} \rightarrow \mathbf{P}^{1}$ is absolutely simple. Let $\tilde{C}$ denote the (good) mod 3 reduction of the fiber $\mathcal{C}_{-1}$, and let $J$ be the jacobian of $\tilde{C}$. Calculating $\# \tilde{C}\left(\mathbf{F}_{3}\right)=1$ and $\# \tilde{C}\left(\mathbf{F}_{9}\right)=15$, we find that the characteristic polynomial of Frobenius for $J$ is $x^{4}-3 x^{3}+7 x^{2}-9 x+9$. A root $\alpha$ generates a quartic field $L$ having a unique nontrivial proper subfield $F$ (of degree 2 ). The $L / F$-conjugate of $\alpha$ is $3 \alpha^{-1}$, and $3 \alpha^{-1} / \alpha$ is not a root of unity, so $\alpha^{n} \notin F$ and $\alpha^{n}$ is of degree 4 for all $n \geq 1$. Hence $J$ is absolutely simple. To deduce from this that the generic fiber of $\mathcal{A} \rightarrow \mathbf{P}^{1}$ is absolutely simple, it suffices to apply the following well-known result twice:

Lemma 2.5. Let $\mathcal{A}$ be an abelian scheme over a discrete valuation ring $R$. If the special fiber is absolutely simple, then so is the generic fiber.

Proof. If the generic fiber is not absolutely simple, then there exists a finite extension $K^{\prime}$ of the fraction field $K$ of $R$ and an isogeny $\mathcal{A} \times{ }_{R} K^{\prime} \rightarrow B \times{ }_{K^{\prime}} C$, where $B$ and $C$ are nontrivial abelian varieties over $K^{\prime}$. Let $R^{\prime}$ be a discrete valuation ring in $L$ containing $R$, and let $k^{\prime} / k$ be the corresponding extension of residue fields. By [ST], the Néron models $\mathcal{B}$ and $\mathcal{C}$ of $B$ and $C$ are abelian schemes over $R^{\prime}$. The universal property of the Néron model extends the isogeny to the Néron models: $\mathcal{A} \times{ }_{R} R^{\prime} \rightarrow \mathcal{B} \times{ }_{R^{\prime}} \mathcal{C}$. Taking special fibers shows that $\mathcal{A} \times{ }_{R} k^{\prime}$ is not simple, so $\mathcal{A} \times_{R} k$ is not absolutely simple.

This completes the proof of Theorem 1.1.

2.5. Review of the Brauer-Manin obstruction. Manin [Ma1] in 1970 introduced what is now called the Brauer-Manin obstruction. Now suppose $X$ is a smooth proper irreducible variety over a number field $k$. Let $\mathbb{A}_{k}$ denote the adèle ring of $k$. If $v$ is a place of $k$, then we may define a "local evaluation pairing"

$$
\mathrm{ev}_{v}: \operatorname{Br}(X) \times X\left(k_{v}\right) \rightarrow \mathbf{Q} / \mathbf{Z},
$$


by letting $\operatorname{ev}_{v}(\alpha, x) \in \mathbf{Q} / \mathbf{Z}$ be the invariant of $i_{x}^{*} \alpha \in \operatorname{Br}\left(k_{v}\right)$ where $i_{x}: \operatorname{Spec} k_{v} \rightarrow X$ corresponds to $x \in X\left(k_{v}\right)$. Given $\alpha$, the properness of $X$ implies that the map $x \mapsto \mathrm{ev}_{v}(\alpha, x)$ is zero for almost all $v$, so by summing over all $v$ we obtain a global pairing

$$
\text { ev }: \operatorname{Br}(X) \times X\left(\mathbb{A}_{k}\right) \rightarrow \mathbf{Q} / \mathbf{Z},
$$

continuous in the second argument, such that $\operatorname{ev}(\alpha, x)=0$ if $\alpha$ comes from $\operatorname{Br}(k)$ or if $x \in X(k)$. One says that there is a Brauer-Manin obstruction for $X$ if for every $x \in X\left(\mathbb{A}_{k}\right)$ there exists $\alpha \in \operatorname{Br}(X)$ with $\operatorname{ev}(\alpha, x) \neq 0$.

It is conjectured that for any smooth, proper, (geometrically) rational surface $X$ over a number field $k$, the Brauer-Manin obstruction is the only obstruction to the Hasse principle; i.e., that if $X(k)=\emptyset$, it is because

$$
\left\{x \in X\left(\mathbb{A}_{k}\right): \operatorname{ev}(\alpha, x)=0 \text { for all } \alpha \in \operatorname{Br}(X)\right\}=\emptyset .
$$

This appeared as a question in [CTS], and as a conjecture in [CTKS], once many examples were available. Any rational surface over $k$ is $k$-birational either to a conic bundle over a conic, or to a Del Pezzo surface of degree $d, 1 \leq d \leq 9$. Del Pezzo surfaces of degree at least 5 satisfy the Hasse principle. The Del Pezzo surfaces of degree 4 are the smooth intersections of two quadrics in $\mathbf{P}^{4}$, and those of degree 3 are the smooth cubic surfaces in $\mathbf{P}^{3}$. There exists theoretical evidence for the conjecture in the case of conic bundles and Del Pezzo surfaces of degree 4, and numerical evidence for diagonal cubic surfaces.

In another direction, Skorobogatov $[\mathrm{Sk}]$ recently gave an example of a bielliptic surface over $\mathbf{Q}$ for which the Brauer-Manin obstruction is not the only one. He and Harari [Ha2], [HS] have discovered that the new obstruction in this and some other examples can be explained by nonabelian unramified covers.

2.6. The evaluation pairing and the Cassels-Tate pairing. It is possible to define the Cassels-Tate pairing in terms of the pairing ev defined above. Suppose $X$ and $Y$ are torsors of an abelian variety $A$ over $k$, representing elements of $\amalg(A)$. We may identify $A$ with $\mathbf{P i c}_{X / k}^{0}$, and then $Y$ corresponds to an element of $\mathrm{H}^{1}\left(k, \operatorname{Pic}^{0} \bar{X}\right)$, which may be mapped to an element $\beta \in \mathrm{H}^{1}(k, \operatorname{Pic} \bar{X})$. The Leray spectral sequence [Mi1, III.1.18(a)] for $X \rightarrow$ Spec $k$ gives rise to an exact sequence

$$
\operatorname{ker}(\operatorname{Br}(X) \rightarrow \operatorname{Br}(\bar{X})) \longrightarrow \mathrm{H}^{1}(k, \operatorname{Pic} \bar{X}) \longrightarrow \mathrm{H}^{3}\left(k, \mathbf{G}_{m}\right)=0
$$

the last equality holding because $k$ is a number field. We pick $x \in X\left(\mathbb{A}_{k}\right)$, and pick $\alpha \in \operatorname{Br}(X)$ mapping to $\beta$ in the sequence above. Then the Cassels-Tate pairing $\langle X, Y\rangle$ equals ev $(\alpha, x)$, which does not depend on the choices made. (See Theorem 41.24 in [Ma2] for the case of genus 1 curves, or the "homogeneous space definition" of the pairing in [PS].)

2.7. The Brauer-Manin obstruction for $\mathcal{X}$. It is conjectured in [CT, Conj. 2] that the Brauer-Manin obstruction to the existence of zero-cycles of degree 1 (the definition is an obvious extension of the one given for rational points) on smooth projective irreducible varieties over number fields is the only one. In this section we show that the nonexistence of zero-cycles of degree 1 on our variety $\mathcal{X}$ can indeed be explained by this obstruction; moreover it can be explained by the obstruction attached to a single element of $\operatorname{Br}(\mathcal{X})$.

Lemma 2.6. Let $k$ be a number field, and let $C$ be a smooth projective geometrically integral curve over $k$. Then $\mathrm{H}^{3}\left(k(C), \mathbf{G}_{m}\right)=0$. 
Proof. If $F$ is any field of characteristic zero, $\mu_{\infty}$ denotes the group of roots of unity in $\bar{F}$, and $Q:=\bar{F}^{*} / \mu_{\infty}$, then $Q$ is uniquely divisible, so $\mathrm{H}^{i}(F, Q)=0$ for $i>0$, and we deduce that $\mathrm{H}^{3}\left(F, \mathbf{G}_{m}\right)=\mathrm{H}^{3}\left(F, \mu_{\infty}\right)=\mathrm{H}^{3}(F, \mathbf{Q} / \mathbf{Z}(1))$.

For real places $v$ of the number field $k$, let $k_{v}^{\prime}:=k_{v} \cap \bar{k} \subset \bar{k}_{v}$ denote the corresponding real closure of $k$. Theorem $1^{\prime}$ of [Ja] states that $\mathrm{H}^{3}(k(C), \mathbf{Q} / \mathbf{Z}(r)) \cong$ $\bigoplus_{v \text { real }} \mathrm{H}^{3}\left(k_{v}^{\prime}(C), \mathbf{Q} / \mathbf{Z}(r)\right)$ for $r \neq 2$. Taking $r=1$, we find that $\mathrm{H}^{3}\left(k(C), \mathbf{G}_{m}\right) \cong$ $\bigoplus_{v \text { real }} \mathrm{H}^{3}\left(k_{v}^{\prime}(C), \mathbf{G}_{m}\right)$.

For $v$ real, let $G_{v}^{\prime}=\operatorname{Gal}\left(\bar{k}(C) / k_{v}^{\prime}(C)\right) \cong \operatorname{Gal}\left(\bar{k} / k_{v}^{\prime}\right) \cong \mathbf{Z} / 2$. The spectral sequence

$$
E_{2}^{p, q}:=\mathrm{H}^{p}\left(G_{v}^{\prime}, \mathrm{H}^{q}\left(\bar{k}(C), \mathbf{G}_{m}\right)\right) \Longrightarrow \mathrm{H}^{p+q}\left(k_{v}^{\prime}(C), \mathbf{G}_{m}\right)
$$

together with the fact (Théorème 1.1 in $[\mathrm{Gr} 2]$ ) that $\mathrm{H}^{q}\left(\bar{k}(C), \mathbf{G}_{m}\right)=0$ for $q>0$ implies that

$$
\mathrm{H}^{3}\left(k_{v}^{\prime}(C), \mathbf{G}_{m}\right)=\mathrm{H}^{3}\left(G_{v}^{\prime}, \bar{k}(C)^{*}\right) .
$$

By periodicity, the latter is $\mathrm{H}^{1}\left(G_{v}^{\prime}, \bar{k}(C)^{*}\right)$, which is zero by Hilbert's Theorem 90.

Corollary 2.7. Let $k$ be a number field, and let $C$ be a smooth projective geometrically integral curve over $k$. Then $\lim _{\mathrm{H} \text { ét }}^{3}\left(V, \mathbf{G}_{m}\right)=0$, where the direct limit is over the dense open subschemes $V$ of $C$, with respect to the restriction maps.

Proof. The direct limit equals $\mathrm{H}^{3}\left(k(C), \mathbf{G}_{m}\right)$, by a result of Grothendieck. See Corollaire 5.9 in SGA 4 VII (Exposé VII: Site et Topos étale d'un schéma, par A. Grothendieck) [SGA4], or Lemma 1.16 and Remark 1.17(a) in [Mi1].

Remark. With more work one can show that if $U \subset C$ is an affine open subset, then $\mathrm{H}_{\text {ét }}^{3}\left(U, \mathbf{G}_{m}\right)$ is finite, but not necessarily zero. Moreover, there exists a dense open subset $U \subset C$ such that $\mathrm{H}_{\text {ét }}^{3}\left(U^{\prime}, \mathbf{G}_{m}\right)=0$ for all open subsets $U^{\prime} \subseteq U$. On the other hand, $\mathrm{H}_{\text {ét }}^{3}\left(C, \mathbf{G}_{m}\right)$ is always infinite.

We now return to our situation, with $\mathcal{C}$ and $U$ as in Section 2.2 and $\mathcal{X}$ as in Section 2.4. Let $V \subseteq U$ be a dense open subset. Recall that $\mathcal{X}_{V}=\mathbf{P i c}_{\mathcal{C}_{V} / V}^{1}$. Let $\iota: \mathcal{C}_{V} \rightarrow \mathcal{X}_{V}$ be the natural inclusion of $V$-schemes. We obtain a commutative diagram of exact sequences of étale sheaves over $V$

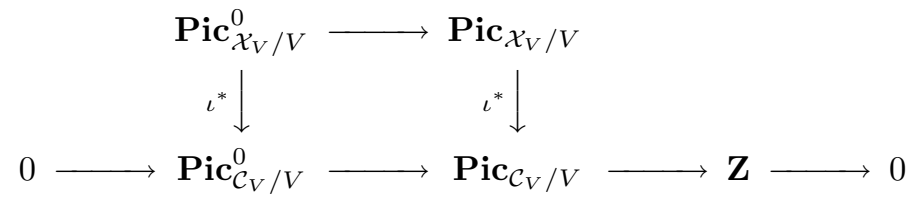

and an argument similar to the one of Proposition 6.9 on p. 118 of [MFK] shows that the first vertical map is an isomorphism. Taking cohomology we obtain:

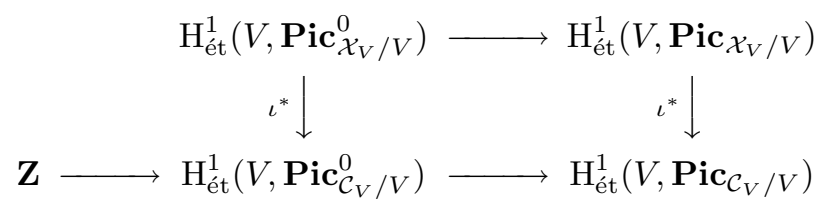

The image of $1 \in \mathbf{Z}$ in $\mathrm{H}_{\text {ét }}^{1}\left(V, \mathbf{P i c}_{\mathcal{C}_{V} / V}^{0}\right)$ is the class $\gamma$ of the torsor $\mathcal{X}_{V}$ of $\mathbf{P i c}_{\mathcal{C}_{V} / V}^{0}$. (The analogous result for the generic fibers, with $V$ replaced by its generic point $\eta:=\operatorname{Spec} \mathbf{Q}(t)$, follows from a cochain calculation in Galois cohomology. The 
claim for $V$ follows, using functoriality and applying the injectivity of $\mathrm{H}_{\text {ét }}^{1}(V, \mathcal{B}) \rightarrow$ $\mathrm{H}^{1}\left(\eta, \mathcal{B}_{\eta}\right)$ for abelian $V$-schemes $\mathcal{B}$ to the case $\mathcal{B}=\mathbf{P i c}_{\mathcal{C}_{V} / V}^{0}$.)

Let $\beta$ be the image of $\left(\iota^{*}\right)^{-1}(\gamma)$ under $\mathrm{H}_{\text {ét }}^{1}\left(V, \mathbf{P i c}_{\mathcal{X}_{V} / V}^{0}\right) \rightarrow \mathrm{H}_{\text {ét }}^{1}\left(V, \mathbf{P i c}_{\mathcal{X}_{V} / V}\right)$. Then $\beta$ maps to 0 in $\mathrm{H}_{\text {ét }}^{1}\left(V, \mathbf{P i c}_{\mathcal{C}_{V} / V}\right)$. The Leray spectral sequences for $\pi_{\mathcal{X}_{V}}$ : $\mathcal{X}_{V} \rightarrow V$ and $\pi_{\mathcal{C}_{V}}: \mathcal{C}_{V} \rightarrow V$ yield a commutative diagram of exact sequences:

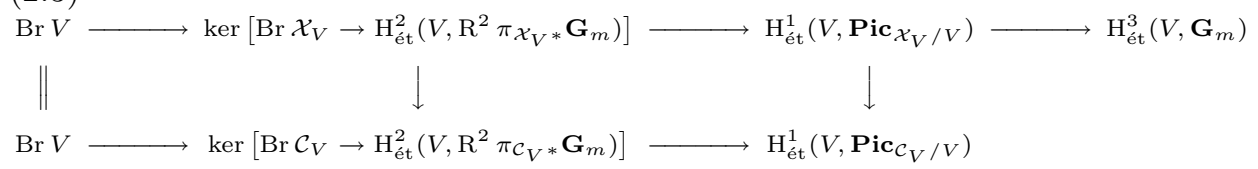

By Corollary 2.7, after shrinking $V$ and restricting if necessary, we may assume that $\beta \in \mathrm{H}_{\text {ét }}^{1}\left(V, \mathbf{P i c}_{\mathcal{X}_{V} / V}\right)$ maps to zero in $\mathrm{H}_{\text {ét }}^{3}\left(V, \mathbf{G}_{m}\right)$, so that $\beta$ lifts leftwards to an element $\alpha \in \operatorname{Br} \mathcal{X}_{V}$. Since $\beta$ maps to 0 in $\mathrm{H}_{\text {ét }}^{1}\left(V, \mathbf{P i c}_{\mathcal{C}_{V} / V}\right)$, we may adjust $\alpha$ by an element in the image of $\operatorname{Br} V$ if necessary, to assume that $\alpha$ restricts to 0 in $\operatorname{Br} \mathcal{C}_{V}$.

Suppose that $t \in V\left(\mathbf{Q}_{p}\right)$ for some finite or infinite prime $p$, and consider the fiber $\mathcal{X}_{t}$, which is a torsor of an abelian variety over $\mathbf{Q}_{p}$. The restriction of $\gamma$ to an element $\gamma_{t}$ of $\mathrm{H}^{1}\left(\mathbf{Q}_{p}, \mathbf{P} \mathbf{i c}_{\mathcal{X}_{t} / \mathbf{Q}_{p}}^{0}\right)$ represents the torsor $\mathbf{P} \mathbf{i c}_{\mathcal{C}_{t} / \mathbf{Q}_{p}}^{1}$, which is trivial by Lemma 2.3. Then the corresponding restriction $\beta_{t} \in \mathrm{H}^{1}\left(\mathbf{Q}_{p}, \mathbf{P} \mathbf{i c}_{\mathcal{X}_{t} / \mathbf{Q}_{p}}\right)$ is 0 , so the analogue of the top row of (2.3) for $\mathcal{X}_{t} \rightarrow \operatorname{Spec} \mathbf{Q}_{p}$ shows that the restriction $\alpha_{t}$ comes from $\operatorname{Br} \mathbf{Q}_{p}$. In particular, $\mathrm{ev}_{p}\left(\alpha_{t}, x\right)$ is a constant function of $x \in \mathcal{X}_{t}\left(\mathbf{Q}_{p}\right)$. For any finite $p$, Lemma 2.2 gives us a point $x \in \mathcal{C}_{t}\left(\mathbf{Q}_{p}\right)$, but $\alpha$ restricts to $0 \in \operatorname{Br} \mathcal{C}_{t}$, so the constant function must be identically zero. Thus for all finite $p, \operatorname{ev}_{p}(\alpha, x)=0$ for all $x \in \mathcal{X}_{V}\left(\mathbf{Q}_{p}\right)$. By [Ha, Théorème 2.1.1], this implies that $\alpha$ extends (uniquely) to an element $\tilde{\alpha} \in \operatorname{Br} \mathcal{X}$.

By continuity, $\operatorname{ev}_{p}(\tilde{\alpha}, x)=0$ for all $x \in \mathcal{X}\left(\mathbf{Q}_{p}\right)$, for any finite $p$. The evaluation of $\tilde{\alpha}$ on an adelic point $x=\left(x_{v}\right) \in \mathcal{X}_{V}\left(\mathcal{A}_{\mathbf{Q}}\right)$ depends only on $x_{\infty}$. The union of the points $x_{\infty} \in \mathcal{X}(\mathbf{R})$ lying above rational points $t \in V(\mathbf{Q})$ is dense in $\mathcal{X}(\mathbf{R})$, and by Section $2.6, \operatorname{ev}(\tilde{\alpha}, x)$ for any such $x$ equals the value of the Cassels-Tate pairing $\left\langle\mathcal{X}_{t}, \mathcal{X}_{t}\right\rangle$, which is $1 / 2$. Hence by continuity ev $(\tilde{\alpha}, x)=1 / 2$ for all $x \in \mathcal{X}\left(\mathcal{A}_{\mathbf{Q}}\right)$, and we deduce a posteriori that $\mathrm{ev}_{\infty}(\tilde{\alpha}, x)=1 / 2$ for all $x \in \mathcal{X}(\mathbf{R})$.

The same argument which showed that $\mathrm{ev}_{p}(\tilde{\alpha}, x)=0$ for all $x \in \mathcal{X}\left(\mathbf{Q}_{p}\right)$ shows that if $k_{v}$ is any nonarchimedean local field containing $\mathbf{Q}$ and $x \in \mathcal{X}\left(k_{v}\right)$, then $\mathrm{ev}_{v}(\tilde{\alpha}, x)=0$.

From the above two paragraphs, we see that for any zero-cycle $z_{v}$ on $\mathcal{X} \times{ }_{\mathbf{Q}} \mathbf{Q}_{v}$, the element $\operatorname{ev}_{v}\left(\tilde{\alpha}, z_{v}\right)$ equals $0 \in \mathbf{Q} / \mathbf{Z}$ if $v$ is a finite place, and equals $d_{v} / 2 \in \mathbf{Q} / \mathbf{Z}$ if $v$ is the real place, where $d_{v}$ is the degree of the zero-cycle $z_{v}$ (over $\mathbf{R}$ ). If the $z_{v}$ for all $v$ arise from a zero-cycle $z$ on $\mathcal{X}$ of odd degree, then summing over $v$ shows that $\operatorname{ev}(\tilde{\alpha}, z)=1 / 2$, which is impossible. By definition, this means that $\tilde{\alpha}$ gives a Brauer-Manin obstruction to the existence of such a zero-cycle $z$.

\section{A family of genus 1 CURVES}

We now prove Theorem 1.2.

3.1. Cubic surfaces violating the Hasse principle. Swinnerton-Dyer [SD1] disproved a conjecture of Mordell [Mo] by constructing a smooth cubic surface $V$ in $\mathbf{P}^{3}$ over $\mathbf{Q}$ "violating the Hasse principle"; i.e., such that $V$ has points over each 
completion of $\mathbf{Q}$, but not over $\mathbf{Q}$. Soon afterwards, Cassels and Guy $[\mathrm{CG}]$ gave the diagonal cubic surface

$$
5 x^{3}+9 y^{3}+10 z^{3}+12 t^{3}=0
$$

violating the Hasse principle.

Manin [Ma1] used the Brauer-Manin obstruction to explain Swinnerton-Dyer's counterexample. (See also [Ma2].) Much later [CTKS] explained the Cassels-Guy example from this point of view, and gave a very explicit algorithm for computing the Brauer-Manin obstruction for diagonal cubic surfaces.

We fix a number field $k$ and a smooth cubic surface $V \subset \mathbf{P}_{k}^{3}$ for which the Hasse principle fails.

3.2. Lefschetz pencils. By 2.5.2 in [Ka], the embedding $V \hookrightarrow \mathbf{P}_{k}^{3}$ is a "Lefschetz embedding." This implies that the statements in the rest of this paragraph hold for a sufficiently generic choice of a $k$-rational line $L$ in the dual projective space $\check{\mathbf{P}}_{k}^{3}$. ( "Sufficiently generic" here means for $L$ corresponding to points outside a certain Zariski closed subset of the Grassmannian.) Let $L^{\prime}$ denote the axis of $L$, that is, the line in $\mathbf{P}_{k}^{3}$ obtained by intersecting two hyperplanes in the family given by $L$. Blowing up $V$ at the scheme-theoretic intersection $V \cap L^{\prime}$ results in a smooth projective variety $V^{\prime}$ over $k$ isomorphic to the reduced subvariety of $V \times L$ whose geometric points are the pairs $(v, H)$ where $v \in V$ is on the hyperplane $H \subset \mathbf{P}_{k}^{3}$ corresponding to a point of $L$. Each fiber of $V^{\prime} \rightarrow L \cong \mathbf{P}_{k}^{1}$ is a proper geometrically integral curve of arithmetic genus 1 , and if it is singular, there is only one singularity and it is a node. The generic fiber is smooth.

3.3. Local points in the pencil. Let $k_{v}$ denote the completion of $k$ at a nontrivial place $v$. Let $\mathcal{O}_{v}$ be the ring of integers, let $\mathfrak{m}_{v}$ be its maximal ideal, and let $\mathbf{F}_{v}=\mathcal{O}_{v} / \mathfrak{m}_{v}$.

Variants of the following lemma have appeared in various places in the literature (e.g. [CTSSD], [CT, 2.1]). The key point is that all the geometric fibers of $V^{\prime} \rightarrow \mathbf{P}^{1}$ are integral.

Lemma 3.1. There exists a finite set $S$ of places of $k$ such that for any $v \notin S$ and any finite extension $K$ of $k_{v}$ the map $V^{\prime}(K) \rightarrow \mathbf{P}^{1}(K)$ is surjective.

Proof. The morphism $f: V^{\prime} \rightarrow \mathbf{P}_{k}^{1}$ is proper and flat. Combining Theorem 11.1.1 and Theorem 12.2.4(viii) of [EGA IV], we see that there exists a finite set $S$ of places of $k$, with associated ring of $S$-integers $\mathcal{O}$, such that $f$ extends to a proper flat morphism $f^{\prime}: \mathcal{V}^{\prime} \rightarrow \mathbf{P}_{\mathcal{O}}^{1}$, all fibers of which are geometrically integral. We may assume that $S$ contains all the real places and none of the complex places. The desired surjectivity is automatic at complex places, so it remains to prove the surjectivity for finite $v \notin S$.

Let $v$ be a place not in $S$, let $K / k_{v}$ be a finite extension with associated valuation $w$, let $\mathcal{O}_{w}$ be the ring of integers of $K$ and let $\mathbf{F}_{w}$ be the residue field. Let $q=\# \mathbf{F}_{w}$. If $t \in \mathbf{P}^{1}\left(\mathcal{O}_{w}\right)=\mathbf{P}^{1}(K)$, then the special fiber of the fiber $\mathcal{V}_{t}^{\prime}$ is a geometrically integral, proper curve of arithmetic genus 1. The number of smooth $\mathbf{F}_{w}$-points on this special fiber is at least $(\sqrt{q}-1)^{2}>0$ (Hasse) if the special fiber is smooth, and is equal to $q-1, q$, or $q+1$ if not; in any case there is at least one. Hensel's Lemma then shows that the generic fiber of $\mathcal{V}_{t}^{\prime}$ has a $K$-point, as desired.

Let $U^{\prime}$ be the largest open subscheme of $\mathbf{P}_{k}^{1}$ over which $V^{\prime}$ is smooth. 
Lemma 3.2. For each completion $k_{v}$ of $k$, the image of $V^{\prime}\left(k_{v}\right) \rightarrow \mathbf{P}^{1}\left(k_{v}\right)$ contains a nonempty open subset $W_{v}$ (in the v-adic topology).

Proof. Recall that $V\left(k_{v}\right) \neq \emptyset$ by choice of $V$. Since $V^{\prime}$ and $V$ are birational smooth projective varieties, $V^{\prime}\left(k_{v}\right) \neq \emptyset$ too, and in fact $V^{\prime}\left(k_{v}\right)$ is Zariski dense in $V^{\prime}$. In particular we can find $P \in V^{\prime}\left(k_{v}\right)$ mapping into $U^{\prime}$. The image of $V^{\prime}\left(k_{v}\right) \rightarrow \mathbf{P}^{1}\left(k_{v}\right)$ will then contain a neighborhood of the image of $P$.

We may choose $S$ as in Lemma 3.1 to contain no complex places. For $v \in S$ choose $W_{v}$ as in Lemma 3.2. We may assume that there exists $v \in S$ for which $W_{v} \subset U^{\prime}\left(k_{v}\right)$. Then the fibers $V_{t}^{\prime}$ for $t \in W_{v}$ are smooth.

\subsection{Base change of the pencil.}

Lemma 3.3. Let $S$ be a finite set of noncomplex places of $k$, let $T$ be a finite set of closed points of $\mathbf{P}_{k}^{1}$, and for each $v \in S$, let $W_{v}$ be a nonempty open subset of $\mathbf{P}^{1}\left(k_{v}\right)$. Then there exists a nonconstant $k$-morphism $f: \mathbf{P}_{k}^{1} \rightarrow \mathbf{P}_{k}^{1}$ such that $f$ is smooth (i.e., unramified) above the points of $T$, and $f\left(\mathbf{P}^{1}\left(k_{v}\right)\right) \subseteq W_{v}$ for all $v \in S$.

Proof. First we show that for each $v \in S$, we can find a nonconstant $f_{v}$ defined over $k_{v}$ such that $f_{v}\left(\mathbf{P}^{1}\left(k_{v}\right)\right) \subseteq W_{v}$. Choose an affine point $\lambda \in W_{v}$. If $v$ is real, we may take $f_{v}(x)=\lambda+\left(x^{2}+n\right)^{-1}$ for $n \gg 0$. If $v$ is finite, then we may choose $g \in k_{v}(x)$ such that $g$ maps $\{0,1, \infty\}$ to $\lambda$, and then let $f_{v}(x)=g\left(x^{n}\right)$ for $n=q^{k}(q-1)$ where $q=\# \mathbf{F}_{v}$ and $k \gg 0$.

Preceding each $f_{v}$ with an arbitrary nonconstant rational function of appropriate degree, we may assume that $\operatorname{deg} f_{v}$ is the same for all $v \in S$. Let $B_{d}$ denote the open affine subset of $\left(a_{0}, \ldots, a_{d}, b_{0}, \ldots, b_{d}\right) \in \mathbf{A}^{2 d+2}$ for which the homogeneous polynomials $\sum a_{i} X^{i} Y^{d-i}$ and $\sum b_{i} X^{i} Y^{d-i}$ have no nontrivial common factor. We have a morphism $B_{d} \times \mathbf{P}^{1} \rightarrow \mathbf{P}^{1}$ which constructs the rational function of degree $d$ which is the quotient of the two polynomials, and then evaluates it at the point in $\mathbf{P}^{1}$. Choose $b_{v} \in B_{d}\left(k_{v}\right)$ representing $f_{v}$. By compactness of $\mathbf{P}^{1}\left(k_{v}\right)$ in the $v$-adic topology, any point of $B_{d}\left(k_{v}\right)$ sufficiently close to $b_{v}$ represents a rational function still mapping $\mathbf{P}^{1}\left(k_{v}\right)$ into $W_{v}$.

Weak approximation gives us a point $b \in B_{d}(k)$ close enough $v$-adically to $b_{v}$ for each $v \in S$ so that the corresponding rational function $f$ over $k$ maps $\mathbf{P}^{1}\left(k_{v}\right)$ into $W_{v}$ for $v \in S$. Moreover $f$ will be smooth above the points of $T$ provided that $b$ avoids a certain closed subset of $B_{d}$ of positive codimension, so this is easily arranged.

Apply Lemma 3.3 to obtain $f$ for the $S$ and the $W_{v}$ at the end of Section 3.3, and with $T=\mathbf{P}_{k}^{1} \backslash U^{\prime}$. We let $\pi_{\mathcal{X}}: \mathcal{X} \rightarrow \mathbf{P}_{k}^{1}$ be the base extension of $V^{\prime} \rightarrow \mathbf{P}_{k}^{1}$ by $f$, so that the following is a cartesian square:

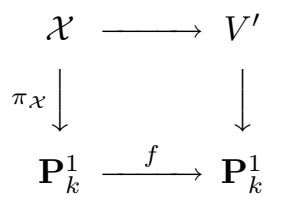

Each factor is smooth over $k$, and above each point of the lower right $\mathbf{P}_{k}^{1}$ at least one of the two factors is smooth by choice of $T$, so $\mathcal{X}$ is smooth over $k$. The generic fiber of $V^{\prime} \rightarrow \mathbf{P}_{k}^{1}$ is geometrically integral, so $\mathcal{X}$ is geometrically integral. Since $V^{\prime}$ is projective over $k$, so is $\mathcal{X}$. 
Let $U=f^{-1}\left(U^{\prime}\right)$. By choice of $f, f\left(\mathbf{P}^{1}(k)\right) \subset U^{\prime}$. Hence $U$ is an open subscheme of $\mathbf{P}_{k}^{1}$ containing $\mathbf{P}^{1}(k)$, and $\mathcal{X}_{U} \rightarrow U$ is a proper smooth family of geometrically integral curves of genus 1 . We may construct $\mathcal{E}$ as the minimal proper regular model of the jacobian of the generic fiber of $\mathcal{X} \rightarrow \mathbf{P}_{k}^{1}$. Then $\mathcal{E}_{U} \cong \mathbf{P i c}_{\mathcal{X}_{U} / U}^{0}$ is an abelian $U$-scheme.

If $v$ is a place of $k$, and $t \in \mathbf{P}^{1}\left(k_{v}\right)$, then $\mathcal{X}_{t} \cong V_{f(t)}^{\prime}$ has a $k_{v}$-point by choice of $f$. The property of having a rational point is a birational invariant of smooth projective varieties, and $V(k)=\emptyset$, so $V^{\prime}(k)=\emptyset$. Since $\mathcal{X}$ maps to $V^{\prime}, \mathcal{X}(k)=\emptyset$ too.

It remains to show that the $j$-invariant of $\pi_{\mathcal{X}}: \mathcal{X} \rightarrow \mathbf{P}_{k}^{1}$ is nonconstant, or equivalently that the $j$-invariant of $\pi: V^{\prime} \rightarrow \mathbf{P}_{k}^{1}$ is nonconstant. Euler-Poincaré characteristic computations [BPV, Prop. (11.4), p. 97] show that the fibration $V^{\prime} \rightarrow \mathbf{P}^{1}$ has exactly 12 nodal geometric fibers. Thus the $j$-invariant of the generic fiber has poles on $\mathbf{P}^{1}$, so it cannot be constant.

3.5. Generic Cassels-Tate pairing. Let $k$ be a number field, let $U \subset \mathbf{P}_{k}^{1}$ be a dense open subscheme of $\mathbf{P}_{k}^{1}$, and let $\mathcal{A}$ be an abelian $U$-scheme. Assume that $\mathcal{A} \rightarrow U$ is projective, so that the dual abelian scheme $\mathcal{A}^{\prime} \rightarrow U$ exists [Gr1]. Now let $\mathcal{X}_{U}$ be an $\mathcal{A}$-torsor over $U$ such that each fiber $\mathcal{X}_{t}$ for $t \in U(k)$ represents a nonzero element of $\amalg\left(\mathcal{A}_{t}\right)$. Is it then automatic that there exists an $\mathcal{A}^{\prime}$-torsor $\mathcal{Y}_{U}$ over $U$ (or at least over some dense open subscheme) such that for all $t \in U(k)$, (the class of) $\mathcal{Y}_{t}$ is in $\amalg\left(\mathcal{A}_{t}^{\prime}\right)$ and the Cassels-Tate pairing $\left\langle\mathcal{X}_{t}, \mathcal{Y}_{t}\right\rangle$ gives a nonzero value in $\mathbf{Q} / \mathbf{Z}$ which does not depend on $t$ ? The special case where the families $\mathcal{A}$ and $\mathcal{X}$ are split is the well-known conjecture that the Cassels-Tate pairing is nondegenerate.

For our first example, the $\mathcal{X}_{U}$ in Section 2 built out of the $\mathbf{P i c}^{1}$ of the relative curve of genus 2 , we can identify $\mathcal{A}$ with $\mathcal{A}^{\prime}$ and take $\mathcal{Y}_{U}=\mathcal{X}_{U}$, because we showed $\left\langle\mathcal{X}_{t}, \mathcal{X}_{t}\right\rangle=1 / 2 \in \mathbf{Q} / \mathbf{Z}$ for all $t \in U(\mathbf{Q})$.

For the second example, the one from the Lefschetz pencil in a cubic surface $V$ over $k$, we can construct $\mathcal{Y}_{U}$, if we assume (as is conjectured) that the failure of the Hasse principle for $V$ is due to a Brauer-Manin obstruction, as we now explain.

The cokernel of $\operatorname{Br}(k) \rightarrow \operatorname{Br}(V)$ for a cubic surface $V$ is finite, but need not be cyclic; see [SD2] for the possibilities. Nevertheless one has the following:

Lemma 3.4. Let $V$ be a smooth cubic surface in $\mathbf{P}^{3}$ over a number field $k$. Assume that the Hasse principle for $V$ fails, and fails because of a Brauer-Manin obstruction for $V$. Then there exists $\alpha \in \operatorname{Br}(V)$ such that $\mathrm{ev}(\alpha, x)$ is a nonzero constant independent of $x \in V\left(\mathbb{A}_{k}\right)$.

Proof. Let $G$ be the cokernel of $\operatorname{Br}(k) \rightarrow \operatorname{Br}(V)$, and let $\hat{G}=\operatorname{Hom}(G, \mathbf{Q} / \mathbf{Z})$. These are finite, and are given the discrete topology. Let $S$ be the image of the continuous map $\phi: V\left(\mathbb{A}_{k}\right) \rightarrow \hat{G}$ induced by ev.

We claim that

$$
x, y, z \in S \Longrightarrow y+z-x \in S .
$$

Because $\phi$ is continuous and $V$ is smooth, given $x, y, z \in S$, we may choose $P=$ $\left(P_{v}\right) \in V\left(\mathbb{A}_{k}\right)$ with $\phi(P)=x$ and similarly $Q$ and $R$ giving $y$ and $z$, so that for each $v, P_{v}, Q_{v}$ and $R_{v}$ are not collinear, and the plane through them intersects $V$ in a nonsingular genus 1 curve $C_{v}$. Applying Riemann-Roch to $C_{v}$ yields a point $T_{v} \in$ $C\left(k_{v}\right)$ linearly equivalent to the divisor $Q_{v}+R_{v}-P_{v}$ on $C_{v}$. As in [Lic], the pairing $\operatorname{Br}\left(C_{v}\right) \times C_{v}\left(k_{v}\right) \rightarrow \operatorname{Br}\left(k_{v}\right) \subseteq \mathbf{Q} / \mathbf{Z}$ extends to a pairing $\operatorname{Br}\left(C_{v}\right) \times \operatorname{Div}\left(C_{v}\right) \rightarrow \mathbf{Q} / \mathbf{Z}$ 
which induces a pairing $\operatorname{Br}\left(C_{v}\right) \times \operatorname{Pic}\left(C_{v}\right) \rightarrow \mathbf{Q} / \mathbf{Z}$. For $\beta \in \operatorname{Br}(V), \operatorname{ev}(\beta, T)$ can be obtained by restricting $\beta$ to $C_{v}$, pairing with $T_{v}$ and summing over $v$. It follows that $\phi(T)=\phi(Q)+\phi(R)-\phi(P)$, so $y+z-x \in S$.

It follows from (3.1) and $S \neq \emptyset$ that $S$ is a coset of a subgroup $H$ of $\hat{G}$. But $S$ cannot be a subgroup, because $0 \in S$ would contradict the existence of a BrauerManin obstruction. Hence we may pick $g \in G \cong \operatorname{Hom}(\hat{G}, \mathbf{Q} / \mathbf{Z})$ annihilating $H$ but not $S$. For any lift $\alpha \in \operatorname{Br}(V)$ of $g, \operatorname{ev}(\alpha, x)$ is a nonzero constant for $x \in V\left(\mathbb{A}_{k}\right)$.

Remarks. 1. Let $Y$ denote the Grassmannian of lines in $\mathbf{P}^{3}$ over $k$. Using correspondences and using the triviality of $\operatorname{Br}(Y) / \operatorname{Br}(k)$, one can prove

$$
x, y \in S \Longrightarrow-x-y \in S,
$$

which improves (3.1). Thus $\alpha$ may be taken so that its image in $\operatorname{Br}(V) / \operatorname{Br}(k)$ has order 3. (This follows also from the calculation of possibilities for $\operatorname{Br}(V) / \operatorname{Br}(k)$ in [SD2], together with Corollary 1 of [SD2], which forces $\operatorname{Br}(V) / \operatorname{Br}(k)$ to have odd order.) Class field theory shows that $3 \operatorname{Br}(k)=\operatorname{Br}(k)$, and it follows formally that the order 3 element of $\operatorname{Br}(V) / \operatorname{Br}(k)$ can be lifted to an order 3 element of $\operatorname{Br}(V)$.

2. One can prove a similar result, namely $x, y, z \in S \Longrightarrow-x-y-z \in S$, for Del Pezzo surfaces $V$ of degree 4 (smooth intersections of two quadrics in $\mathbf{P}^{4}$ ). In this case $\operatorname{Br}(V) / \operatorname{Br}(k)$ is killed by 2. (See p. 178 in [Ma2] or the proof of Proposition 3.18 in [CTSSD].) If there is a Brauer-Manin obstruction, then it can be explained by a single element $\alpha \in \operatorname{Br}(V)$ of order 2. (If $k$ has real places, $2 \operatorname{Br}(k) \neq \operatorname{Br}(k)$, so the argument in the previous remark needs to be modified in order to prove this; one must make use of the assumption that $V\left(k_{v}\right) \neq \emptyset$ for all real places $v$.)

3. Suppose that $V$ is a Del Pezzo surface of degree 4 over $k$ as above, with a Brauer-Manin obstruction given by $\alpha \in \operatorname{Br}(V)$ of order 2. A result of Amer [Am] and Brumer [Br] then implies that $V(L)=\emptyset$ for any finite extension $L$ of odd degree over $k$. According to the conjecture in Section 2.5, there should be a Brauer-Manin obstruction for $V \times{ }_{k} L$. Does the same $\alpha$ yield an obstruction over $L$ ?

4. If one replaces $V$ by a smooth cubic surface in the previous question, and the Amer-Brumer result by the conjecture that the existence of a point of degree prime to 3 on $V$ implies the existence of a $k$-point, then one is led to ask the analogous question for $V$, for extensions $L$ with $\operatorname{gcd}([L: k], 3)=1$.

5. There is a geometric application of the Amer-Brumer result that leads to a question about Brauer-Manin obstructions for Weil restriction of scalars. Let $L$ be a finite separable field extension of $k$, and let $W$ be a quasi-projective variety over $L$. If $R:=\operatorname{Res}_{L / k} W$ is the Weil restriction of scalars, then there is a natural $L$-morphism $R_{L}:=R \times_{k} L \rightarrow W$. Now let $V$ be a Del Pezzo surface of degree 4 over $k$, and apply the above to $W:=V_{L}=V \times_{k} L$. We obtain a morphism $R_{L} \rightarrow V_{L}$. If $[L: k]$ is odd, then application of the Amer-Brumer result for $V$ to the extension of function fields $L(R) / k(R)$ yields a $k$-rational map $R \rightarrow V$. A Brauer-Manin obstruction to the existence of a $k$-point on $V$ can be pulled back to obtain a Brauer-Manin obstruction for $R$ over $k$. Hence we are led to the following question, to which a positive answer would imply a positive answer to the question in remark 3 above:

Let $L / K$ be a finite extension of number fields. Let $V$ be a smooth projective geometrically integral variety over $L$, and let $R=\operatorname{Res}_{L / k}(V)$. It is clear that $V$ has 
points over all completions of $L$ if and only if $R$ has points over all completions of $k$. Suppose that this is the case. Then is it true that there exists a Brauer-Manin obstruction for $V / L$ if and only if there exists a Brauer-Manin obstruction for $R / k$ ?

If it is true that the Brauer-Manin obstruction is the only one for geometrically rational varieties, then the answer must be yes for such varieties. For arbitrary $V$, one direction can be proved without too much work: if there is a BrauerManin obstruction for $V / L$, then because of the natural map $R_{L} \rightarrow V$ described above, there is a Brauer-Manin obstruction for $R_{L} / L$, and compatibility of the corestriction map $\operatorname{Br}\left(R_{L}\right) \rightarrow \operatorname{Br}(R)$ with pullback shows that there is a BrauerManin obstruction for $R / k$.

We return to the notation of earlier subsections of Section 3. In particular, $V$ is a smooth cubic surface violating the Hasse principle. By our assumption (the conjecture), there is a Brauer-Manin obstruction. Recall that we are trying to show that the lack of rational points on $V$ can be explained by the Cassels-Tate pairing.

Choose $\alpha$ as in Lemma 3.4. Let $g$ be the composite morphism $\mathcal{X} \rightarrow V^{\prime} \rightarrow V$, and define $\alpha^{\prime}:=g^{*} \alpha \in \operatorname{Br}(\mathcal{X})$, which gives a Brauer-Manin obstruction for $\mathcal{X}$. We take $U$ to be the largest open subscheme of $\mathbf{P}_{k}^{1}$ over which $\pi_{\mathcal{X}}: \mathcal{X} \rightarrow \mathbf{P}_{k}^{1}$ is smooth, and let $\mathcal{X}_{U}:=\pi_{X}^{-1}(U)$ as usual. We have an exact sequence of étale sheaves of groups

$$
0 \rightarrow \mathbf{P i c}_{\mathcal{X}_{U} / U}^{0} \rightarrow \mathbf{P i c}_{\mathcal{X}_{U} / U} \rightarrow \mathbf{Z} \rightarrow 0
$$

and $\mathrm{H}_{\text {ét }}^{1}(U, \mathbf{Z})=0$ by Proposition 3.6(ii) in $[\mathrm{Ar}]$. This explains the bottom row of

$$
\operatorname{Br}\left(\mathcal{X}_{U}\right)
$$

$$
\mathrm{H}_{\text {ét }}^{1}\left(U, \mathbf{P i c}_{\mathcal{X}_{U} / U}^{0}\right) \longrightarrow \mathrm{H}_{\text {ét }}^{1}\left(U, \mathbf{P i c}_{\mathcal{X}_{U} / U}\right) \longrightarrow 0 \text {. }
$$

The vertical map is the homomorphism

$$
\operatorname{ker}\left[\mathrm{H}_{\text {ét }}^{2}\left(\mathcal{X}_{U}, \mathbf{G}_{m}\right) \rightarrow \mathrm{H}_{\text {ét }}^{0}\left(U, \mathrm{R}^{2} \pi_{*} \mathbf{G}_{m}\right)\right] \longrightarrow \mathrm{H}_{\text {ét }}^{1}\left(U, \mathrm{R}^{1} \pi_{*} \mathbf{G}_{m}\right)
$$

from the Leray spectral sequence for $\pi: \mathcal{X}_{U} \rightarrow U$, since the sheaf $\mathrm{R}^{1} \pi_{*} \mathbf{G}_{m}$ can be identified with $\mathbf{P i c}_{\mathcal{X}_{U} / U}$ by [BLR, p. 203], and $\mathrm{R}^{2} \pi_{*} \mathbf{G}_{m}=0$ by Corollaire 3.2 in [Gr2].

We restrict $\alpha^{\prime}$ to an element of $\operatorname{Br}\left(\mathcal{X}_{U}\right)$, map it downwards in (3.2) to obtain $\beta \in \mathrm{H}_{\text {ét }}^{1}\left(U, \mathbf{P i c}_{\mathcal{X}_{U} / U}\right)$, and lift leftwards to obtain $\gamma \in \mathrm{H}_{\text {ét }}^{1}\left(U, \mathbf{P i c}_{\mathcal{X}_{U} / U}^{0}\right)$. By [Mi1, III.4.7], $\gamma$ is the class of some torsor $\mathcal{Y}_{U}$ for $\mathbf{P i c}_{\mathcal{X}_{U} / U}^{0}=\mathcal{E}_{U}$ over $U$.

Proposition 3.5. With notation as above, assuming that there is a Brauer-Manin obstruction for $V$, the class of $\mathcal{Y}_{t}$ is in $\amalg\left(\mathcal{E}_{t}\right)$ for all $t \in U(k)$, and the Cassels-Tate pairing $\left\langle\mathcal{X}_{t}, \mathcal{Y}_{t}\right\rangle \in \mathbf{Q} / \mathbf{Z}$ is a nonzero constant independent of $t$.

Proof. Suppose $t \in U(k)$. If $\epsilon$ is a cohomology class of an étale sheaf on $U$ (or $\mathcal{X}_{U}$ ), let $\epsilon_{t}$ denote the restriction to $t$ (resp. $\left.\mathcal{X}_{t}\right)$. We have a diagram

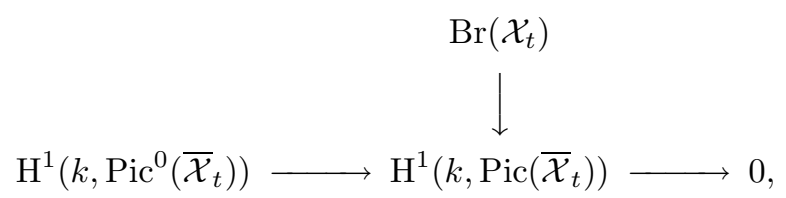

analogous to (3.2), in which the restrictions $\alpha_{t}^{\prime}$ on the top and $\gamma_{t}$ on the left map to $\beta_{t}$. The class of the fiber $\mathcal{Y}_{t}$ equals $\gamma_{t}$. 
Then $\operatorname{ev}\left(\alpha_{t}^{\prime}, x\right)=\operatorname{ev}(\alpha, g(x))$ is a nonzero constant independent of $x \in \mathcal{X}_{t}\left(\mathbb{A}_{k}\right)$ (and also independent of $t$ ). The former is a sum of local functions $\mathrm{ev}_{v}\left(\alpha_{t}^{\prime}, x_{v}\right)$ of independent arguments, so each of these must be constant. Let $E=\mathcal{X}_{t} \times_{k} k_{v}$, which is an elliptic curve over $k_{v}$, since $\mathcal{X}_{t}\left(k_{v}\right) \neq \emptyset$. Let $\bar{E}=E \times_{k_{v}} \bar{k}_{v}$. A point in $\operatorname{Pic}^{0}(E)\left(k_{v}\right)$ is represented by a difference of two points in $E\left(k_{v}\right)$, and the pairing $\operatorname{Br}(E) \times \operatorname{Pic}(E) \rightarrow \operatorname{Br}\left(k_{v}\right)$ induced by evaluation is compatible with the pairing $\mathrm{H}^{1}\left(k_{v}, \operatorname{Pic} \bar{E}\right) \times \operatorname{Pic}^{0}(E) \rightarrow \operatorname{Br}\left(k_{v}\right) \hookrightarrow \mathbf{Q} / \mathbf{Z}$ [Lic], so the image $\beta_{E}$ of $\beta_{t}$ in $\mathrm{H}^{1}\left(k_{v}, \mathrm{Pic} \bar{E}\right)$ is in the kernel of the latter pairing. It follows from Tate local duality (or a result of Witt if $v$ is real) that $\beta_{E}=0$.

In the diagram analogous to (3.3), but over $k_{v}$ instead of $k$, the bottom surjection is now an isomorphism, since the first map in the exact sequence

$$
\mathrm{H}^{0}\left(k_{v}, \operatorname{Pic}(\bar{E})\right) \rightarrow \mathbf{Z} \rightarrow \mathrm{H}^{1}\left(k_{v}, \operatorname{Pic}^{0}(\bar{E})\right) \rightarrow \mathrm{H}^{1}\left(k_{v}, \operatorname{Pic}(\bar{E})\right) \rightarrow 0
$$

is surjective. Hence the image of $\gamma_{t}$ in $\mathrm{H}^{1}\left(k_{v}, \operatorname{Pic}^{0}(\bar{E})\right)$, which maps to $\beta_{E}=0$, must itself be zero. Thus $\gamma_{t} \in \amalg\left(\mathcal{E}_{t}\right)$. By Section 2.6, $\left\langle\mathcal{X}_{t}, \mathcal{Y}_{t}\right\rangle=\operatorname{ev}\left(\alpha_{t}^{\prime}, x\right)$ for any $x \in \mathcal{X}_{t}\left(\mathbb{A}_{k}\right)$, and the right hand side is independent of $t$.

\section{ACKNOWLEDGEMENTS}

We thank Sir Peter Swinnerton-Dyer for some discussions about cubic surfaces, in particular for a suggestion which led to the proof of Lemma 3.4. We also thank Qing Liu for directing us to [Liu].

\section{REFERENCES}

[Am] M. Amer, Quadratische Formen über Funktionenkörpern, Dissertation, Mainz, 1976.

[Ar] M. Artin, Faisceaux constructibles. Cohomologie d'une courbe algébrique, Exposé IX, pp. 1-42 in Théorie des topos et cohomologie étale des schémas (SGA 4). Tome 3, Lecture Notes in Math., 305, Springer, Berlin, 1973. MR 50:7132

[BPV] W. Barth, C. Peters, and A. Van de Ven, Compact complex surfaces, Ergebnisse der Mathematik und ihrer Grenzgebiete, 3. Folge, Band 4, Springer-Verlag, Berlin, 1984. MR 86c:32026

[BLR] S. Bosch, W. Lütkebohmert, and M. Raynaud, Néron models, Ergebnisse der Mathematik und ihrer Grenzgebiete, 3. Folge, Band 21, Springer-Verlag, Berlin, 1990. MR 91i:14034

[Br] A. Brumer, Remarques sur les couples de formes quadratiques, C. R. Acad. Sci. Paris 286, série A (1978), 679-681. MR 58:16502

[CG] J. W. S. Cassels and M. J. T. Guy, On the Hasse principle for cubic surfaces, Mathematika 13 (1966), 111-120. MR 35:2841

[CT] J.-L. Colliot-Thélène, The Hasse principle in a pencil of algebraic varieties, Contemporary Math. 210 (1998), 19-39. MR 98g:11075

[CTS] J.-L. Colliot-Thélène et J.-J. Sansuc, La descente sur les variétés rationnelles, in Journées de Géométrie Algébrique d'Angers, Juillet 1979/Algebraic Geometry, Angers, 1979, 223-237, Sijthoff \& Noordhoff, Alphen aan den Rijn, 1980. MR 82d:14016

[CTKS] J.-L. Colliot-Thélène, D. Kanevsky, et J.-J. Sansuc, Arithmétique des surfaces cubiques diagonales, pp. 1-108 in Diophantine approximation and transcendence theory (Bonn, 1985), Lecture Notes in Math., 1290, Springer, Berlin, 1987. MR 89g:11051

[CTSSD] J.-L. Colliot-Thélène, J.-J. Sansuc, and Sir Peter Swinnerton-Dyer, Intersections of two quadrics and Châtelet surfaces. I, J. Reine Angew. Math. 373 (1987), 37-107. MR 88m: $11045 \mathrm{a}$

[DM] P. Deligne and D. Mumford, The irreducibility of the space of curves of given genus, Inst. Hautes Études Sci. Publ. Math., No. 36, 1969, 75-109. MR 41:6850 
[EGA IV] A. Grothendieck, Éléments de géométrie algébrique, rédigés avec la collaboration de J. Dieudonné. IV. Étude locale des schémas et des morphismes de schémas (Troisième Partie), Inst. Hautes Études Sci. Publ. Math., No. 28, 1966. MR 36:178

[Gr1] A. Grothendieck, Technique de descente et théorèmes d'existence en géométrie algébrique. V. Les schémas de Picard: théorèmes d'existence, in Séminaire Bourbaki, Vol. 7, Exp. 232, 143-161, Soc. Math. France, Paris, 1995. CMP 98:09

[Gr2] A. Grothendieck, Le groupe de Brauer III: exemples et compléments, pp. 88-188 in Dix exposés sur la cohomologie des schémas, Adv. Stud. Pure Math., 3, North-Holland, Amsterdam, 1968. MR 39:5586c

[Ha] D. Harari, Méthode des fibrations et obstruction de Manin, Duke Math. J. 75 (1994), 221-260. MR 95j:11056

[Ha2] D. Harari, Weak approximation and non-abelian fundamental groups, preprint, October 1998.

[HS] D. Harari and A. N. Skorobogatov, Non-abelian cohomology and rational points, preprint, May 1999.

[Ja] U. Jannsen, Principe de Hasse cohomologique, Séminaire de Théorie des Nombres, Paris, 1989-1990, Sinnou David (ed.), Progress in Mathematics 102, 1992, 121-140. MR 99a: 11080

[Ka] N. Katz, Pinceaux de Lefschetz: théorème d'existence, Exposé XVII, pp. 212-253 in Groupes de monodromie en géométrie algébrique (SGA 7 II), Lecture Notes in Math., 340, Springer, Berlin, 1973. MR 50:7135

[Lic] S. Lichtenbaum, Duality theorems for curves over $p$-adic fields, Invent. math. 7 (1969), 120-136. MR 39:4158

[Lin] C.-E. Lind, Untersuchungen über die rationalen Punkte der ebenen kubischen Kurven vom Geschlecht Eins, Thesis, University of Uppsala, 1940.

[Liu] Q. Liu, Modèles entiers d'une courbe hyperelliptique sur un corps de valuation discrète, Trans. Amer. Math. Soc. 348 (1996), no. 11, 4577-4610. MR 97h:11062

[Ma1] Yu. I. Manin, Le groupe de Brauer-Grothendieck en géométrie diophantienne, in Actes du Congrès International des Mathématiciens (Nice, 1970), Tome 1, 401-411, Gauthier-Villars, Paris, 1971. MR 55:356

[Ma2] Yu. I. Manin, Cubic forms, Translated from the Russian by M. Hazewinkel, Second edition, North-Holland, Amsterdam, 1986. MR 87d:11037

[Mi1] J. Milne, Étale cohomology, Princeton Univ. Press, Princeton, N.J., 1980. MR 81j:14002

[Mi2] J. Milne, Arithmetic duality theorems, Perspective in Mathematics 1, Academic Press, 1986. MR 88e: 14028

[Mo] L. J. Mordell, Rational points on cubic surfaces, Publ. Math. Debrecen 1 (1949), 1-6. MR 11:82h

[MFK] D. Mumford, J. Fogarty, and F. Kirwan, Geometric invariant theory. Third edition, Ergebnisse der Mathematik und ihrer Grenzgebiete, 2. Folge, Band 34, Springer-Verlag, Berlin, 1994. MR 95m:14012

[PS] B. Poonen and M. Stoll, The Cassels-Tate pairing on polarized abelian varieties, to appear in Ann. of Math.

[Re] H. Reichardt, Einige im Kleinen überall lösbare, im Grossen unlösbare diophantische Gleichungen, J. Reine Angew. Math. 184 (1942), 12-18. MR 5:141c

[Sc] C. Scheiderer, Real and étale Cohomology, Lecture Notes in Math., Vol. 1588, Springer, Berlin, 1994. MR 96c:14018

[ST] J.-P. Serre and J. Tate, Good reduction of abelian varieties, Ann. of Math. (2) 88 (1968), 492-517. MR 38:4488

[SGA4] SGA 4, Théorie des topos et cohomologie étale des schémas, Tome 1. Théorie des topos. Séminaire de Géométrie Algébrique du Bois-Marie 1963-1964. Dirigé par M. Artin, A. Grothendieck, et J. L. Verdier. Avec la collaboration de N. Bourbaki, P. Deligne et B. Saint-Donat. Lecture Notes in Mathematics, Vol. 269, Springer-Verlag, Berlin-New York, 1972.

[Sk] A. N. Skorobogatov, Beyond the Manin obstruction, Invent. Math. 135 (1999), no. 2, 399-424. CMP 99:07

[SD1] H. P. F. Swinnerton-Dyer, Two special cubic surfaces, Mathematika 9 (1962), 54-56. MR 25:3413 
[SD2] Sir Peter Swinnerton-Dyer, The Brauer group of cubic surfaces, Math. Proc. Cambridge Philos. Soc. 113 (1993), no. 3, 449-460. MR 94a:14038

[vGY] J. van Geel and V. Yanchevskiǐ, Indices of hyperelliptic curves over p-adic fields, Manuscripta Math. 96 (1998), 317-333. MR 99e:11084

C.N.R.S., Mathématiques, Bâtiment 425, Université de Paris-Sud, F-91405 Orsay, FRANCE

E-mail address: colliot@math.u-psud.fr

Department of Mathematics, University of California, Berkeley, California 94720 3840

E-mail address: poonen@math.berkeley.edu 